\title{
POSITIVE UNSTABLE PERIODIC SOLUTIONS FOR SUPERLINEAR PARABOLIC EQUATIONS
}

\author{
NORIMICHI HIRANO AND NORIKO MIZOGUCHI
}

(Communicated by Barbara Lee Keyfitz)

ABSTRACT. In this paper, we are concerned with a superlinear parabolic equation

$$
\begin{cases}\frac{\partial u}{\partial t}-\Delta u=u^{p}+h(t, x), & (t, x) \in \mathbf{R}_{+} \times \Omega, \\ u=0, & (t, x) \in \mathbf{R}_{+} \times \partial \Omega, \\ u>0, & (t, x) \in \mathbf{R}_{+} \times \partial \Omega,\end{cases}
$$

where $\Omega \subset \mathbf{R}^{N}$ is a bounded domain with smooth boundary $\partial \Omega, h$ is $T$ periodic with respect to the first variable, and $1<p<\frac{N+2}{N-2}$ if $N \geq 3$ and $1<p<+\infty$ if $N \leq 2$. It is shown that there exist a stable and an unstable positive $T$-periodic solution for this problem if $h$ is sufficiently small in $L^{\infty}$.

\section{INTRODUCTION}

Let $\Omega$ be a bounded domain in $\mathbb{R}^{N}$ with smooth boundary $\partial \Omega$ and $h: \mathbf{R}_{+} \times$ $\bar{\Omega} \rightarrow \mathbf{R}$ be a bounded function which is $T$-periodic with respect to the first variable. In this paper, we are concerned with stable and unstable $T$-periodic solutions for a semilinear parabolic equation

$$
\begin{cases}\frac{\partial u}{\partial t}-\Delta u=u^{p}+h(t, x) & \text { in } \mathbf{R}_{+} \times \Omega, \\ u=0 & \text { in } \mathbf{R}_{+} \times \partial \Omega, \\ u(t)=u(t+T) & \text { in } \bar{\Omega}_{1}, u>0 \text { in } \mathbf{R}_{+} \times \Omega,\end{cases}
$$

where $1<p<(N+2) /(N-2)$ if $N \geq 3$ and $1<p<+\infty$ if $N \leq 2$.

In the recent decade, many authors have studied the existence and multiplicity of periodic solutions for semilinear parabolic boundary value problems of the form

$$
\begin{cases}\frac{\partial u}{\partial t}-\Delta u=g(t, x, u) & \text { in } \mathbf{R}_{+} \times \boldsymbol{\Omega}, \\ u=0 & \text { on } \mathbf{R}_{+} \times \partial \boldsymbol{\Omega},\end{cases}
$$

where $g: \mathbf{R}_{+} \times \Omega \times \mathbf{R} \times \mathbf{R}$ is $T$-periodic with respect to the first variable. One can find extensive references in [2] for the existence of periodic solutions of $(*)$.

Received by the editors March 31, 1993 and, in revised form, August 19, 1993.

1991 Mathematics Subject Classification. Primary 35K20, 35B10.

Key words and phrases. Parabolic nonlinear problem, periodic solutions, stable solutions, unstable solutions. 
On the other hand, the stability of periodic solutions of a semilinear parabolic boundary value problem has been studied by Alikakos, Hess, and Matano [1], Hess [12], Hirsch [15], and the authors. The method employed in these works in the sub- and supersolution method. If there exist a subsolution $\underline{u}$ and a supersolution $\bar{u}$ of the problem (*) satisfying $\underline{u}<\bar{u}$, one can find a stable periodic solution of the problem (cf. Dancer and Hess [6]). A few sufficient conditions for the existence of sub- and supersolutions for $(*)$ is known in case that $g$ has a sublinear growth (cf. Hess [12]). The existence of unstable solutions for (*) is considered in Hirano [13] in the case that $g$ has an asymptotically linear nonlinearity.

On the other hand, it seems that only a few results are known when the nonlinearity is superlinear. Esteban [7] obtained the existence of a positive periodic solution of the problem $(*)$ in the case of $g(t, x, \xi)=m(t) \xi^{p}$ with $1<p<(3 N+8) /(3 N-4)$. She improved her results in [8] to the case that $1<p<N /(N-2)$. It is proved in Ôtani [16] that there exists a periodic solution for $(\mathrm{P})$ if $h$ is sufficiently small with $1<p<(N+2) /(N-2)$.

In this paper, we show that the problem $(\mathrm{P})$ possesses at least one stable and one unstable solution for small $h$. To show the existence of solutions of $(\mathrm{P})$, we calculate the Leray-Schauder degree of an associated compact mapping by using the method employed in Esteban [7]. The stability and instability of solutions of $(P)$ are investigated in $\S 3$.

\section{EXISTENCE OF PERIODIC SOLUTIONS FOR (P)}

In this section, we first describe our theorem and then evaluate the LeraySchauder degree to show the existence of positive periodic solutions. Throughout the rest of this paper, we fix $q>N+1$. For simplicity, put $Q_{T}=(0, T) \times \Omega$. Let

$$
W^{1,2, r}\left(Q_{T}\right)=\left\{u: Q_{T} \rightarrow \mathbf{R} \mid u_{x_{i} x_{j}}, u_{x_{i}}, u_{t} \in L^{r}\left(Q_{T}\right) \text { for } 1 \leq i, j \leq N\right\}
$$

and

$$
C^{1,2}\left(\bar{Q}_{T}\right)=\left\{u: \bar{Q}_{T} \rightarrow \mathbf{R} \mid U_{x_{i} x_{j}}, u_{x_{i}}, u_{t} \in C\left(\bar{Q}_{T}\right) \text { for } 1 \leq i, j \leq N\right\},
$$

where $u_{x_{i} x_{j}}=\partial^{2} u / \partial x_{i} \partial x_{j}, u_{x_{i}}=\partial u / \partial x_{i}$, and $u_{t}=\partial u / \partial t$. We set $E=$ $W^{1,2, q}\left(Q_{T}\right)$ and denote by $\|\cdot\|,\|\cdot\|_{q}$, and $\langle\langle\cdot, \cdot\rangle\rangle$ the norms of $E, L^{q}\left(Q_{T}\right)$, and the inner-product of $L^{2}\left(Q_{T}\right)$, respectively. $B_{r}(v)$ stands for the closed ball in $E$ with radius $r$ centered at $v$.

A solution $u$ of $(\mathrm{P})$ is said to be stable if for given $\varepsilon>0$, there exists $\delta>0$ such that for each $v_{0} \in L^{q}(\Omega)$ with $\left|v_{0}-u(0)\right|_{q}<\delta$, there exists the global solution $v$ for the initial value problem

$$
\begin{cases}\frac{\partial v}{\partial t}-\Delta v=v^{p}+h(t, x) & \text { in }(0, \infty) \times \Omega, \\ v=0 & \text { on }(0, \infty) \times \partial \Omega, \\ v(0)=v_{0} & \text { in } \Omega,\end{cases}
$$

and it holds that $|v(t)-u(t)|_{q}<\varepsilon$ for all $t>0$. Here $|\cdot|_{q}$ stands for the norm of $L^{q}(\Omega)$. A $T$-periodic solution $u$ is called unstable if $u$ is not stable. The purpose of this paper is to prove the following result. 
Theorem. Let $h \in L^{\infty}\left(\mathbf{R}_{+} \times \Omega\right)$ be nonnegative and T-periodic with respect to the first variable. If $\|h\|_{L^{\infty}}$ is sufficiently small, then there exist at least one stable and one unstable solution of $(\mathrm{P})$ in $E$.

We denote by $\lambda_{1}$ and $\varphi_{1}$ the first eigenvalue of $-\Delta$ in $\Omega$ with the Dirichlet boundary respectively. Let $L=\partial / \partial t-\Delta$ with domain

$$
D(L)=\{u \in E: u(T)=u(0) \text { in } \Omega\} .
$$

A compact operator $K: E \rightarrow E$ can be defined by $L^{-1}$ since there is uniquely $u \in E$ satisfying $L u=v$ for any $v \in E$ arbitrarily given. Let $f: \mathbf{R} \rightarrow \mathbf{R}$ be the extension of $\xi^{p}$ defined by $f(\xi)=0$ for all $\xi<0$, and let $g(t, x, \xi)=$ $f(\xi)+h(t, x)$. The solutions for (P) are fixed points of the operator $K \circ g$ from $E$ into itself. We first investigate the Leray-Schauder degree of $I-K \circ g$ near 0 and then in a large ball in $E$. We denote by $\operatorname{deg}(F, U, v)$ the degree of compact operator $F$ on an open set $U$ with respect to $v$.

Lemma 1. For given $c>0$, there exist positive numbers $r<c$ and $\delta$ such that for each $h \in L^{\infty}\left(Q_{T}\right)$ with $\|h\|_{\infty} \leq \delta$,

$$
\operatorname{deg}\left(I-K \circ g, B_{r}(0), 0\right)=1 .
$$

Proof. According to the standard regularity theory, there exists $C>0$ such that

$$
\|u\| \leq C\|g(t, x, u)\|_{q}
$$

for any solution $u \in D(L)$ for $L u=s g(t, x, u)$ with $s \in[0,1]$. Let $c>0$. Take $0<\rho<1$ satisfying $\rho / C_{1}<c$ and $\xi^{p+1} \leq \frac{\lambda_{1}}{2} \xi^{2}$ for all $\xi \in \mathbf{R}$ with $|\xi| \leq \rho$, where $C_{1}$ is the constant of the embedding of $E$ into $C\left(\bar{Q}_{T}\right)$. We put $r=\rho / C_{1}$. Let $\delta>0$ such that

$$
\frac{\lambda_{1}}{2}\left(\frac{1}{C} r-\delta\right)^{q}-\delta r>0
$$

and $\|h\|_{\infty} \leq \delta$. Suppose that there is a solution $u \in D(L) \cap \partial B_{r}(0)$ for $L u=$ $s g(t, x, u)$ for some $s \in[0,1]$. Then noting that $p q>2$, we have

$$
\begin{aligned}
0 & =\left\langle\left\langle u_{t}-\Delta u-s g(t, x, u), u\right\rangle\right\rangle \\
& =\int_{0}^{T} \int_{\Omega}\left(|\nabla u|^{2}-s u^{p+1}-s h u\right) d x d t \\
& \geq \frac{\lambda_{1}}{2} \int_{0}^{T} \int_{\Omega} u^{p q} d x d t-\int_{0}^{T} \int_{\Omega} h u d x d t \\
& \geq \frac{\lambda_{1}}{C}\left(\frac{1}{C}\|u\|-\|h\|_{\infty}\right)^{q}-\|h\|_{\infty}\|u\|>0 .
\end{aligned}
$$

This contradiction implies the nonexistence of solutions of $L u=s g(t, x, u)$ on $\partial B_{r}(0)$. From the homotopy invariance of the Leray-Schauder degree, it follows that

$$
\operatorname{deg}\left(I-K \circ g, B_{r}(0), 0\right)=\operatorname{deg}\left(I, B_{r}(0), 0\right)=1 .
$$

The following lemma is proved by Esteban for $1<p<(N+2) / N$ in [7] and for $1<p<N /(N-2)$ in [8] in the case that the right-hand side of $(\mathrm{P})$ is $m(t, x) u^{p}$. The proof of the following lemma is almost exactly as in [7] and [8] (cf. [10]). 
Lemma 2. For each $h \in L^{\infty}\left(Q_{T}\right)$, there exists $R>r$ such that

$$
\operatorname{deg}\left(I-K \circ g, B_{R}(0), 0\right)=0 .
$$

Proof. The proof of this lemma based on the same idea as in the proof of Theorem 4 and the alternative proof of Proposition 12 of [7]. Then we will only sketch the proof. We first get the uniform estimate for solutions of $(P)$ in $L^{\infty}\left(Q_{T}\right)$. Indeed, on the contrary, assume that there is a sequence $\left\{u_{n}\right\}$ of solutions of $(\mathrm{P})$ such that $M_{n}=\sup _{Q_{T}} u_{n}$ diverges to $+\infty$. Let $\left(t_{n}, P_{n}\right) \in Q_{T}$ with $M_{n}=u_{n}\left(t_{n}, P_{n}\right)$ and $\left\{\left(t_{n}, P_{n}\right)\right\}$ converge to some $(\bar{t}, P)$. In case of $(\bar{t}, P) \in Q_{T}$, put

$$
\lambda_{n}^{2 /(p-1)} M_{n}=1, \quad y=\frac{x-P_{n}}{\lambda_{n}}, \quad \tau=\frac{t-t_{n}}{\lambda_{n}^{2}}, \quad v_{n}(\tau, y)=\lambda_{n}^{2 /(p-1)} u_{n}(t, x) .
$$

By the same argument as in [7], $\left\{v_{n}\right\}$ converges to some $v \in C^{1,2}\left(\mathbf{R} \times \mathbf{R}^{N}\right)$ in $C^{1,2}\left([-R, R] \times \bar{B}_{R}(0)\right)$ for any $R>0$. Then $v$ satisfies $v_{t}-\Delta v=c v^{p}$ in $\mathbf{R} \times \mathbf{R}^{N}$ and $v(0,0)=1$. On the other hand, multiplying (P) with $u$ replaced by $u_{n}$ by $\left(u_{n}\right)_{t}$ and integrating on $Q_{T}$, it follows that

$$
\int_{0}^{T} \int_{\Omega}\left(u_{n}\right)_{t}^{2} d x d t \leq \int_{\omega} \int_{\Omega} h^{2} d x d t
$$

Therefore, for any $R>0$ and sufficiently large $n$, we have

$$
\int_{-R}^{R} \int_{B_{R}(0)}\left(v_{n}\right)_{\tau}^{2} d y d \tau \leq \lambda_{n}^{4 /(p-1)-N+2} \int_{0}^{T} \int_{\Omega} h^{2} d x d t \rightarrow 0 \quad \text { as } n \rightarrow \infty,
$$

and hence $v_{t} \equiv 0$ in $\mathbf{R} \times \mathbf{R}^{N}$. According to a Liouville theorem in [9], $v$ is identically zero, which contradicts $v(0,0)=1$. In case that $(\bar{t}, P) \in[0, T] \times$ $\partial \Omega \cup\{0, T\} \times \Omega$, we obtain a contradiction by taking the same process as in [7] using that $v_{t} \equiv 0$. The above argument shows that there exists $L_{0}>0$ such that any solution $u$ of $(\mathrm{P})$ satisfies $\|u\|_{L^{\infty}}<L_{0}$. We can now complete the proof by the same argument as in the proof of Theorem 4 of [7]. That is, we define a family of compact operators $\left\{T_{s}\right\}$ from $E$ into itself as follows: $T_{s} u=v$ if and only if $v$ is a solution of the problem

$$
\begin{cases}\frac{\partial v}{\partial t}-\Delta v=v^{p}+s u+s+h(t, x), & (t, x) \in \mathbf{R}_{+} \times \Omega, \\ v=0, & (t, x) \in \mathbf{R}_{+} \times \partial \Omega, \\ v(t)=v(t+T) & \text { in } \bar{\Omega}, v>0 \text { in } \mathbf{R}_{+} \times \Omega .\end{cases}
$$

From the $L^{\infty}$ estimate above, we have that there exists $C(s)>0$ such that if $u$ is a fixed point of $T_{s}$, then $\|u\| \leq C(s)$. On the other hand, we can see that $T_{s}$ has no fixed point if $s$ is sufficiently large. Then we have that there exists $R>0$ such that $\|u\| \leq R$ if $u$ is a fixed point of $T_{s}$ for some $s>0$. Then since $\operatorname{deg}\left(I-T_{s}, B_{R}(0), 0\right)=0$ for $s$ sufficiently large, the assertion follows from the homotopy invariance of degree.

\section{STABILITY AND INSTABILITY OF PERIODIC SOlutions}

We discuss the stability and the instability for solutions of $(\mathrm{P})$ in the present section. Let $u$ be a $T$-periodic solution for $(\mathrm{P})$. Denote by $S(t, s)$ the evolution 
operator for the following problem:

$$
\begin{cases}\frac{d v}{d t}-\Delta v=f^{\prime}(u) v & \text { in }(s, \infty) \times \Omega, \\ v=0 & \text { on }(s, \infty) \times \partial \Omega, \\ v(s)=z & \text { in } \Omega ;\end{cases}
$$

that is, $S(t, s) z=v(t)$. Then nonzero eigenvalues of the periodic map $U(t)=$ $S(t+T, t)$ is independent of $t$ (see [11]). It is known that if $U(t)$ satisfies

$$
\sigma(U(t)) \cap\{\mu:|\mu|>1\} \neq \varnothing,
$$

where $\sigma(A)$ means the set of eigenvalues of a linear operator $A$, then $u$ is unstable (see [11, Theorem 8.1.2]). By the result due to Beltramo and Hess [3], we have that the linear operator $L_{u}=L-f^{\prime}(u)$ with domain $D(L)$ has a unique real principal eigenvalue $\mu_{u}$ with an associated positive eigenfunction $\varphi_{u}$. It is known that $u$ is stable if $\mu_{u}>0$ and $u$ is unstable if $\mu_{u}<0$ (cf. [3, 11]). We use a similar idea employed in [13] to prove the following result.

Lemma 3. Let $u$ be a solution of $(\mathrm{P})$. Then there exists $\rho>0$ satisfying the following conditions:

(i) If $\mu_{u}>0$, then $\operatorname{deg}\left(I-K \circ g, B_{\rho}(u), 0\right)=1$.

(ii) If $\mu_{u}=0$, then $\operatorname{deg}\left(I-K \circ g, B_{\rho}(u), 0\right)=0$.

Proof. Suppose that $\mu_{u}>0$. Then $K \circ f^{\prime}(u)$ has no real eigenvalue larger than or equal to 1 (cf. [3]). Then we have $\operatorname{deg}\left(I-K \circ f^{\prime}(u), B_{r}(0), 0\right)=1$ for any $r>0$ (cf. [5, Theorem 8.10]). From the definition of $\mu_{u}$, we have that

$$
\left\langle\left\langle L v-f^{\prime}(u) v, v\right\rangle\right\rangle \geq \mu_{u}\|v\|_{2}^{2}
$$

for all $v \in E$. Then it is easy to see that there exists $r>0$ such that

$$
\langle\langle L v-g(t, x, v), v\rangle\rangle>0 \quad \text { for all } v \in B_{r}(u) \backslash\{u\} .
$$

This implies that $u$ is an isolated solution of $(\mathbf{P})$ and by the homotopy invariance of degree, we find that

$$
\operatorname{deg}\left(I-K \circ g, B_{\rho}(0), 0\right)=\operatorname{deg}\left(I-K \circ f^{\prime}(u), B_{\rho}(0), 0\right)=1
$$

for some $\rho>0$ sufficiently small. We next prove statement (ii). Define $\widetilde{L}, \widetilde{K}$, and $\tilde{f}$ by $\widetilde{L}(u)=L u+u$ for $u \in D(L), \widetilde{K}=(\widetilde{L})^{-1}$, and $\tilde{f}(\xi)=f(\xi)+\xi$ for $\xi \in \mathbf{R}$. For simplicity, write $\varphi_{u}$ by $\varphi$. Then $\mu_{u}$ is the principal eigenvalue of $\widetilde{L}-\tilde{f}^{\prime}(u)$ and $\varphi$ is an eigenfunction of $\widetilde{L}-\tilde{f}^{\prime}(u)$ corresponding to $\mu_{u}$. We set $E_{1}=\{s \varphi: s \in \mathbf{R}\}$. Then there is a closed subspace $E_{2}$ of $L^{\infty}\left(Q_{T}\right)$ which is invariant under $\widetilde{K} \circ \tilde{f}^{\prime}(u)$ such that $L^{\infty}\left(Q_{T}\right)=E_{1} \oplus E_{2}$. Denoting by $P_{i}$ the projection from $L^{\infty}\left(Q_{T}\right)$ onto $E_{i}$ for $i=1,2, P_{i}$ is a bounded operator, that is, there exists $M>0$ satisfying

$$
\left\|P_{i} v\right\|_{\infty} \leq M\|v\|_{\infty}, \quad i=1,2 \text {, }
$$

for all $v \in L^{\infty}\left(Q_{T}\right)$. Since $\tilde{f}$ is a mapping of class $C^{2}$ from $L^{\infty}\left(Q_{T}\right)$ into itself, the following Taylor expansions hold in $B_{\delta}(0)$ for some $\delta>0$ :

(2) $\tilde{f}^{\prime}(u+v)=\tilde{f}^{\prime}(u)+f^{\prime \prime}(u) v+h_{2}(v), \quad f^{\prime \prime}(u+v)=f^{\prime \prime}(u)+h_{2}(v)$ 
for $v \in B_{\delta}(0)$, where $h_{1}(v) \in o\left(\|v\|_{\infty}^{2}\right), h_{2}(v) \in o\left(\|v\|_{\infty}\right)$, and $h_{3}(v) \in$ $O\left(\|v\|_{\infty}^{p-2}\right)$. Here we choose $\varepsilon>0$ satisfying

$$
\{s \varphi:|s|<\varepsilon\} \times\left\{v \in E_{2}:\|v\|<\varepsilon\right\} \subset B_{\delta}(0)
$$

and put $U_{\varepsilon}(u)=u+\{s \varphi:|s|<\varepsilon\} \times\left\{v \in E_{2}:\|v\|<\varepsilon\right\}$.

Now we show that $\left(f^{\prime \prime}(u) / \tilde{f}^{\prime}(u)\right) \varphi^{2} \notin E_{2}$. From the definition of $f$ and $\tilde{f}$, we have that $f^{\prime \prime}(u) / \tilde{f}^{\prime}(u) \geq 0$ and $\left(f^{\prime \prime}(u) / \tilde{f}^{\prime}(u)\right) \varphi^{2} \in E$. Assume that $\left(f^{\prime \prime}(u) / \tilde{f}^{\prime}(u)\right) \varphi^{2} \in E_{2}$. Then we have that there is $w \in L^{\infty}\left(Q_{T}\right)$ such that

$$
\left.\tilde{L} w-f^{\prime}(u) w=\tilde{f}^{\prime \prime}(u) / \tilde{f}^{\prime}(u)\right) \varphi^{2} .
$$

Since 0 is the principal eigenvalue of $\widetilde{L}-\tilde{f}^{\prime}(u)$, we obtain that there exists a positive function $\tilde{\varphi} \in E$ satisfying

$$
-\tilde{\varphi}_{t}-\Delta \tilde{\varphi}+\tilde{\varphi}-\tilde{f}^{\prime}(u) \tilde{\varphi}=0
$$

by [4]. From (3) and (4), it follows that

$$
\begin{aligned}
0 & <\int_{0}^{T} \int_{\Omega} \frac{f^{\prime \prime}(u)}{\tilde{f}^{\prime}(u)} \varphi^{2} \tilde{\varphi} d x d t \\
& =\int_{0}^{T} \int_{\Omega}\left(w_{t}-\Delta w+w-\tilde{f}^{\prime}(u) w\right) \tilde{\varphi} d x d t \\
& =\int_{0}^{T} \int_{\Omega}\left(-\tilde{\varphi}_{t}-\Delta \tilde{\varphi}+\tilde{\varphi}-\tilde{f}^{\prime}(u) \tilde{\varphi}\right) w d x d t=0 .
\end{aligned}
$$

This contradiction implies that $\left(f^{\prime \prime}(u) / \tilde{f}^{\prime}(u)\right) \notin E_{2}$. Therefore, there exists $r \neq 0$ satisfying

$$
\frac{f^{\prime \prime}(u)}{\tilde{f}^{\prime}(u)} \varphi^{2}-P_{2}\left(\frac{f^{\prime \prime}(u)}{\tilde{f}^{\prime}(u)} \varphi^{2}\right)=r \varphi .
$$

Fix $v \in E_{2}$ with $\|v\|<\varepsilon$, and define

$$
\psi_{v}(s)=\langle\langle\widetilde{L}(u+v+s \varphi)-\tilde{f}(u+v+s \varphi)-h, r \varphi\rangle\rangle
$$

for $s \in \mathbf{R}$ with $|s|<\varepsilon$. Then we have from (1) and (2) that

$$
\begin{gathered}
\psi_{v}(0)=\left\langle\left\langle\tilde{L} v-\tilde{f}^{\prime}(u) v-\frac{f^{\prime \prime}(u)}{2} v^{2}-h_{1}(v), r \varphi\right\rangle\right\rangle, \\
\psi_{v}^{\prime}(0)=-\left\langle\left\langle f^{\prime \prime}(u) v \varphi+h_{2}(v) \varphi, r \varphi\right\rangle\right\rangle,
\end{gathered}
$$

and

$$
\psi_{v}^{\prime \prime}(0)=-\left\langle\left\langle f^{\prime \prime}(u) \varphi^{2}+h_{3}(v) \varphi^{2}, r \varphi\right\rangle\right\rangle .
$$

Consequently, we find

$$
\begin{aligned}
\psi_{v}(s)= & \left\langle\left\langle\tilde{L} v-\tilde{f}^{\prime}(u) v-\frac{f^{\prime \prime}(v)}{2} v^{2}-h_{1}(v), r \varphi\right\rangle\right\rangle \\
& -\left\langle\left\langle f^{\prime \prime}(u) v \varphi+h_{2}(v) \varphi, r \varphi\right\rangle\right\rangle s \\
& -\frac{1}{2}\left\langle\left\langle f^{\prime \prime}(u) \varphi^{2}+h_{3}(v) \varphi^{2}, r \varphi\right\rangle\right\rangle s^{2}+h_{4}(s),
\end{aligned}
$$

where $h_{4}(s) \in o\left(s^{2}\right)$. For $\rho \in[0,1]$, set

$$
g_{\rho}(t, x, \xi)=g(t, x, \xi)+\rho r \tilde{f}^{\prime}(u) \varphi .
$$


We claim that for each $0<\rho \leq 1$, the compact mapping $K \circ g_{\rho}$ has no fixed point in $U_{\varepsilon}(u)$ and that $u$ is the unique fixed point of $K \circ g$ in $U_{\varepsilon}(u)$. Suppose that

$$
L(u+w+\tau \varphi)=g_{\rho}(t, x, u+w+\tau \varphi)
$$

for some $\rho \in[0,1], w \in E_{2}$, and $\tau \in \mathbf{R}$ with $\|w\|,|\tau|<\varepsilon$. Then we get by (1) that

$$
\widetilde{L} w-\tilde{f}^{\prime}(u) w-\frac{f^{\prime \prime}(u)}{2}(w+\tau \varphi)^{2}-h_{1}(w+\tau \varphi)=\rho r \tilde{f}^{\prime}(u) \varphi .
$$

Multiplying this equality by $1 / \tilde{f}^{\prime}(u)$ and projecting both sides of the equality onto $E_{2}$, it follows that

(7) $\tilde{L} w-\tilde{f}^{\prime}(u) w=\frac{\tilde{f}^{\prime}(u)}{2} P_{2}\left(\frac{f^{\prime \prime}(u)}{\tilde{f}^{\prime}(u)}(w+\tau \varphi)^{2}\right)+\tilde{f}^{\prime}(u) P_{2}\left(\frac{h_{1}(w+\tau \varphi)}{\tilde{f}^{\prime}(u)}\right)$

and hence

$$
w=\left(\widetilde{L}-\tilde{f}^{\prime}(u)\right)^{-1}\left(\frac{\tilde{f}^{\prime}(u)}{2} P_{2}\left(\frac{f^{\prime \prime}(u)}{\tilde{f}^{\prime}(u)}(w+\tau \varphi)^{2}\right)+\tilde{f}^{\prime}(u) P_{2}\left(\frac{h_{1}(w+\tau \varphi)}{\tilde{f}^{\prime}(u)}\right)\right) .
$$

From the inequality above, we have that there exists $C_{1}>0$ satisfying

$$
\|w\|_{\infty} \leq C_{1}\left(\|w\|_{\infty}^{2}+\tau^{2}\right)
$$

Since we may choose $\varepsilon$ so small that $C_{1} \varepsilon<1 / 2$, we obtain from the inequality above that

$$
\|w\|_{\infty} \leq 2 C_{1} \tau^{2}
$$

for each $w \in E$ satisfying (6). Then we have from (5), (6), (7), and the equality above that

$$
\begin{aligned}
0 & \leq \rho\left\langle\left\langle\tilde{f}^{\prime}(u) r \varphi, r \varphi\right\rangle\right\rangle=\psi_{w}(\tau) \\
& =-\frac{1}{2}\left\langle\left\langle f^{\prime \prime}(u) \varphi^{2}-\tilde{f}^{\prime}(u) P_{2}\left(\frac{f^{\prime \prime}(u)}{\tilde{f}^{\prime}(u)} \varphi^{2}\right), r \varphi\right\rangle\right\rangle \tau^{2}+h_{5}(\tau) \\
& =-\frac{1}{2}\left\langle\left\langle\tilde{f}^{\prime}(u) r \varphi, r \varphi\right\rangle\right\rangle \tau^{2}+h_{5}(\tau),
\end{aligned}
$$

where $h_{5} \in o\left(\tau^{2}\right)$. Then we have from the inequality above that $w \in E_{2}$ with $\|w\| \leq \varepsilon$ satisfies (6) only when $\rho=0$ and $w=0$. Thus we have shown that $K \circ g_{\rho}$ has no fixed point in $U_{\varepsilon}(u)$ for $1<\rho \leq 1$ and $u$ is the unique fixed point of $K \circ g$ in $U_{\varepsilon}(u)$. Then according to the homotopy invariance of the Leray-Schauder degree, we find that

$$
\operatorname{deg}\left(I-K \circ g, U_{\varepsilon}(u), 0\right)=\operatorname{deg}\left(I-K \circ g_{1}, U_{\varepsilon}(u), 0\right)=0 .
$$

This completes the proof.

Proof of Theorem. By Lemma 1 , there is $r>0$ sufficiently small such that the problem $(\mathrm{P})$ possesses a solution $u_{1}$ in $B_{r}(0)$. We may assume that $0 \leq$ $f^{\prime}(v)<\lambda_{1}$ on $\Omega$ for each $v \in B_{r}(0)$. Letting $\mu_{u 1}$ and $\varphi_{u 1}$ as before, it holds that

$$
\left(\varphi_{u 1}\right)_{t}=\Delta \varphi_{u 1}-f^{\prime}(u) \varphi_{u 1}=\mu_{u 1} \varphi_{u 1}
$$


Assume that $\mu_{u 1} \leq 0$. From this equality and the definition of $\lambda_{1}$, it follows that

$$
\int_{0}^{T} \int_{\Omega} \lambda_{1} \varphi_{1} \varphi_{u 1} d x d t=\int_{0}^{T} \int_{\Omega}\left\{f^{\prime}(u)+\mu_{u 1}\right\} \varphi_{u 1} \varphi_{1} d x d t<\int_{0}^{T} \int_{\Omega} \lambda_{1} \varphi_{u 1} \varphi_{1} d x d t .
$$

This contradiction implies that $\mu_{u 1}>0$, and then $u_{1}$ is stable. On the other hand, taking $R$ sufficiently large, we have by Lemma 2 that

$$
\operatorname{deg}\left(I-K \circ g, B_{R}(0) \backslash B_{r}(0), 0\right)=-1 .
$$

We can see from the proof of Lemma 3 that if $u$ is a solution of $(\mathrm{P})$ in $B_{R}(0)$ satisfying $\mu_{u} \geq 0$, then $u$ is isolated. Then by Lemma 3, we conclude that there exists $u_{2} \in B_{R}(0) \backslash B_{r}(0)$ with $\mu_{u 2}<0$. Therefore, $u_{2}$ is unstable. This completes the proof.

\section{REFERENCES}

1. N. D. Alikakos, P. Hess, and H. Matano, Discrete order preserving semigroups and stability for periodic parabolic differential equations, J. Differential Equations 82 (1989), 322-341.

2. H. Amann, Periodic solutions for semi-linear parabolic equations, Nonlinear Analysis: A Collection of Papers in Honor of Erich Rothe, Academic Press, New York, 1978, pp. 1-29.

3. A Beltramo and P. Hess, On the principal eigenvalue of a periodic-parabolic operator, Comm. Partial Differential Equations 9 (1984), 919-941.

4. A. Castro and A. C. Lazer, Results on periodic solutions of parabolic equations suggested by elliptic theory, Boll. Un. Mat. Ital. B 1 (1982), 1089-1104.

5. K. Deimling, Nonlinear functional analysis, Springer-Verlag, New York, 1985.

6. E. N. Dancer and P. Hess, On stable solutions of quasilinear periodic-parabolic problems, preprint.

7. M. Esteban, On periodic solutions of superlinear parabolic problems, Trans. Amer. Math. Soc. 293 (1986), 171-189.

8. _ A remark on the existence of positive periodic solutions of superlinear parabolic problems, Proc. Amer. Math. Soc. 102 (1988), 131-136.

9. B. Gidas and J. Spruck, Global and local behavior of positive solutions of nonlinear elliptic equations, Comm. Pure. Appl. Math. 34 (1981), 525-598.

10. Y. Giga, $A$ bound for global solutions of semilinear heat equations, Comm. Math. Phys. 103 (1986), 415-421.

11. D. Henry, Geometry theory of semilinear parabolic equations, Lecture Notes in Math., vol. 840, Springer-Verlag, New York, 1981.

12. $\mathrm{P}$. Hess, On positive solutions of semilinear periodic-parabolic problems in infinite-dimensional systems (Kappel-Schappacher, ed.), Lecture Notes in Math., vol. 1076, SpringerVerlag, New York, 1984, pp. 101-114.

13. N. Hirano, Existence of unstable periodic solutions for semilinear parabolic equations, Nonlinear Anal. 23 (1994), 732-744.

14. N. Hirano and N. Mizoguchi, Existence of unstable periodic solutions for semilinear parabolic equations, preprint.

15. M. W. Hirsch, Differential equations and convergence almost everywhere in strongly monotone semiflows, Contemp. Math., vol. 17, Amer. Math. Soc., Providence, RI, 1983, pp. 267-285.

16. M. Ôtani, Nonmonotone perturbations for nonlinear parabolic equations associated with subdifferential operators, periodic problems, J. Differential Equations (1984), 248-273. 
17. J. Prüss, Periodic solutions of semilinear evolution equations, Nonlinear Anal. 3 (1979), 601-612.

18. I. I. Vrabie, Periodic solutions for nonlinear evolution equations in a Banach space, Proc. Amer. Math. Soc. 109 (1990), 635-661.

Department of Mathematics, Faculty of Engineering, Yokohama National UniverSITY, TOKIWADAI, HodOGAYA-KU, YoKoHAMA 156, JAPAN

E-mail address: hirano@math.sci.ymu.ac.jp 